\title{
Mechanism of molecular beam epitaxy growth of GaN nanowires on $\mathrm{Si}(111)$
}

R. K. Debnath, R. Meijers, T. Richter, T. Stoica, R. Calarco, and H. Lüth

Citation: Appl. Phys. Lett. 90, 123117 (2007);

View online: https://doi.org/10.1063/1.2715119

View Table of Contents: http://aip.scitation.org/toc/apl/90/12

Published by the American Institute of Physics

\section{Articles you may be interested in}

From nucleation to growth of catalyst-free GaN nanowires on thin AIN buffer layer

Applied Physics Letters 91, 251902 (2007); 10.1063/1.2817941

VAPOR-LIQUID-SOLID MECHANISM OF SINGLE CRYSTAL GROWTH

Applied Physics Letters 4, 89 (1964); 10.1063/1.1753975

GaN based nanorods for solid state lighting

Journal of Applied Physics 111, 071101 (2012); 10.1063/1.3694674

Nucleation and growth of GaN nanorods on $\mathrm{Si}$ (111) surfaces by plasma-assisted molecular beam epitaxy - The influence of Si- and Mg-doping

Journal of Applied Physics 104, 034309 (2008); 10.1063/1.2953087

Emission color control from blue to red with nanocolumn diameter of InGaN/GaN nanocolumn arrays grown on same substrate

Applied Physics Letters 96, 231104 (2010); 10.1063/1.3443734

A growth diagram for plasma-assisted molecular beam epitaxy of GaN nanocolumns on $\mathrm{Si}(111)$

Journal of Applied Physics 106, 126102 (2009); 10.1063/1.3267151

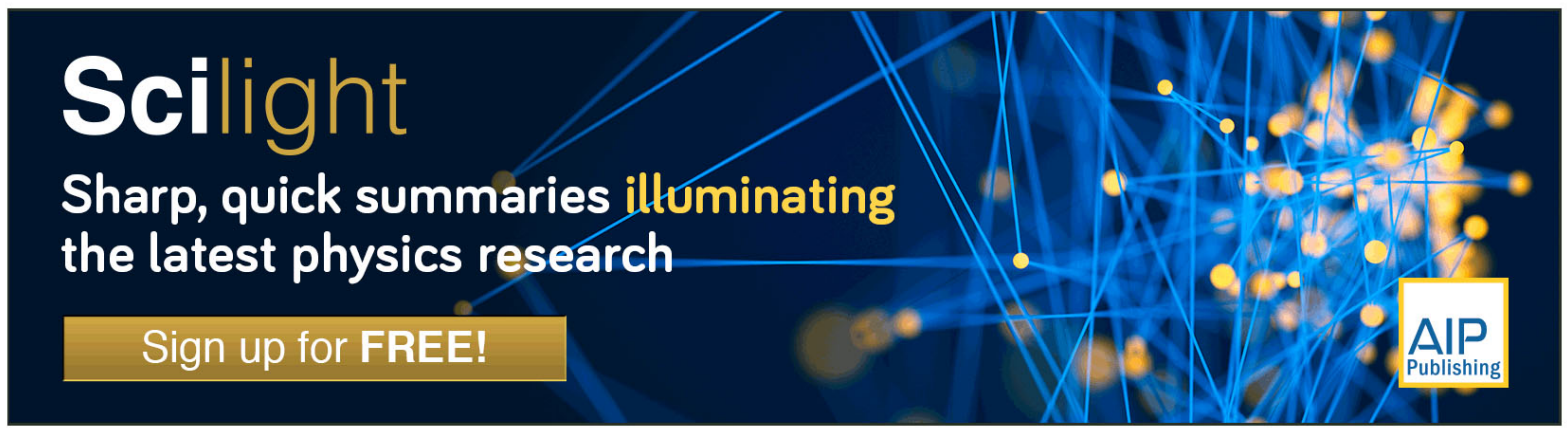




\title{
Mechanism of molecular beam epitaxy growth of GaN nanowires on $\mathrm{Si}(111)$
}

\author{
R. K. Debnath, R. Meijers, T. Richter, T. Stoica, R. Calarco, ${ }^{a)}$ and H. Lüth \\ Institute of Bio- and Nanosystems (IBN-1) and Center of Nanoelectronic Systems for Information \\ Technology (cni), Research Centre Jülich GmbH, D-52425 Jülich, Germany
}

(Received 11 September 2006; accepted 15 February 2007; published online 22 March 2007)

\begin{abstract}
$\mathrm{GaN}$ nanowires have been grown without external catalyst on $\mathrm{Si}(111)$ substrates by plasma-assisted molecular beam epitaxy. Nanowire aspect ratios (length/diameter) of about 250 have been achieved. During the initial stage of the growth, there is a nucleation process in which the number of wires increases and the most probable nucleation diameter of about $10 \mathrm{~nm}$ has been observed, which slowly increases with deposition time. For deposition time longer than the nucleation stage, the nanowire length as a function of diameter monotonically decreases. This phenomenon can be explained by adatom diffusion on the nanowire lateral surface towards the tip. (C) 2007 American Institute of Physics. [DOI: 10.1063/1.2715119]
\end{abstract}

Nanowires (NWs) have attracted much interest in recent time because of their potential for the fabrication of electronic and optoelectronic devices. ${ }^{1-3}$ Different techniques such as metal-organic vapor phase epitaxy, ${ }^{4}$ plasma-assisted molecular beam epitaxy (PAMBE), ${ }^{5,6}$ and chemical beam epitaxy ${ }^{7}$ have been used to grow a variety of semiconductor NWs with extremely high crystalline quality. For instance, GaN NWs grown by molecular beam epitaxy (MBE) show very strong luminescence efficiency. As far as the growth mechanism is concerned, the vapor-liquid-solid (VLS) model $^{8}$ for catalyst-enhanced growth by chemical and physical vapor phase epitaxy has been well established and accepted widely. However, a lot of uncertainty remains on the mechanisms driving the growth of nitride NWs by MBE without any external catalyst. A detailed study is necessary to understand the growth mechanism of these wires.

To develop a model for the growth of NWs, it is very important to take into account the different kinetic adatom processes such as adsorption, desorption, and surface diffusion. Especially adatom diffusion is a key parameter which can have a significant influence on growth rate, material quality, and surface morphology. Most of the research works on NWs, dealing with growth mechanisms, are based on catalyst-induced growth by a metal particle. ${ }^{9,10}$ It has been argued that in some cases the metal particle acts as a collector species rather than a catalyst. ${ }^{11}$ Very recently a comprehensive generalized dynamic model has been proposed by Dubrovskii et al. ${ }^{12,13}$ for MBE growth of III-V NWs driven by Au particles. On the other hand, NW growth without external catalyst is not understood in such detail at present.

The mechanism for the growth of GaN NWs by MBE is still not clear and there are very few investigations ${ }^{14,15}$ dealing with growth mechanism. It has been previously postulated that $\mathrm{Ga}$ droplets serve as a catalyst in a VLS-type growth mechanism. ${ }^{16}$ However, all the reports in literature including the work of Meijers et al. ${ }^{5}$ with ex situ investigations never showed the presence of Ga droplets, neither on the surface nor on the tip of the NWs. It has to be mentioned here that in our experiments after deposition, the sample remains in $\mathrm{N}$ atmosphere till the chamber is pumped down to base pressure, which implies a possible consumption of the

\footnotetext{
${ }^{a)}$ Electronic mail: r.calarco@fz-juelich.de
}

Ga droplets. An in situ investigation of the growth to reveal the presence of $\mathrm{Ga}$ droplets is required to address this issue. In the experiments, GaN NWs have been grown by radio frequency PAMBE on $\mathrm{Si}(111)$. A detailed description of the experimental growth conditions can be found elsewhere. ${ }^{5} \mathrm{~A}$ set of samples was investigated by the scanning electron microscopy (SEM) with respect to width and length of the NWs.

From our understanding of the NW growth without an external catalyst particle, the top surface of nucleated wires acts like an efficient collector of adatoms. In this letter, we tentatively explain the growth by making use of the diffusion-induced (DI) mechanism. The adatoms diffuse to the wire apex from its lateral sides driven by a lower chemical potential at the top surface. This DI mechanism is used to explain the observed dependence of the length with wire diameter for deposition time longer than the nucleation time, but for a complete understanding of the growth including the nucleation process further and more detailed investigations are necessary.

In this letter, we propose an explanation for the growth mechanism of PAMBE-grown GaN NWs based on DI mechanism at different growth stages. A schematic of the different phenomena playing a role during the growth is shown in Fig. 1. In addition to the direct impingement of atoms at the tip of the NW, there is an important contribution to the total NW growth due to the diffusion of adatoms towards the tip on side faces. It is worth noting that we do not have evidence for the formation of a wetting layer in our samples. The thickness of the wetting layer, if any, is below the resolution of the technique used for the investigations shown in this letter. Therefore, we will neglect the wetting layer thickness in comparison with the NW length.

For low and intermediate NW densities, there is a continuous nucleation of wires, whereas the wires already nucleated continue to grow in length and diameter (data not shown here). Towards higher densities, the space for nucleation of other wires drops and the "nucleation rate" approaches zero. From the SEM investigations of the NW formation (images not shown here), it is clear that the NWs seem to grow on the flat steps of the $\mathrm{Si}(111)$ surface without any preferential nucleation sites.

After nucleation, the diameter grows slowly compared to the length resulting in an increase of the aspect ratio. Histo- 


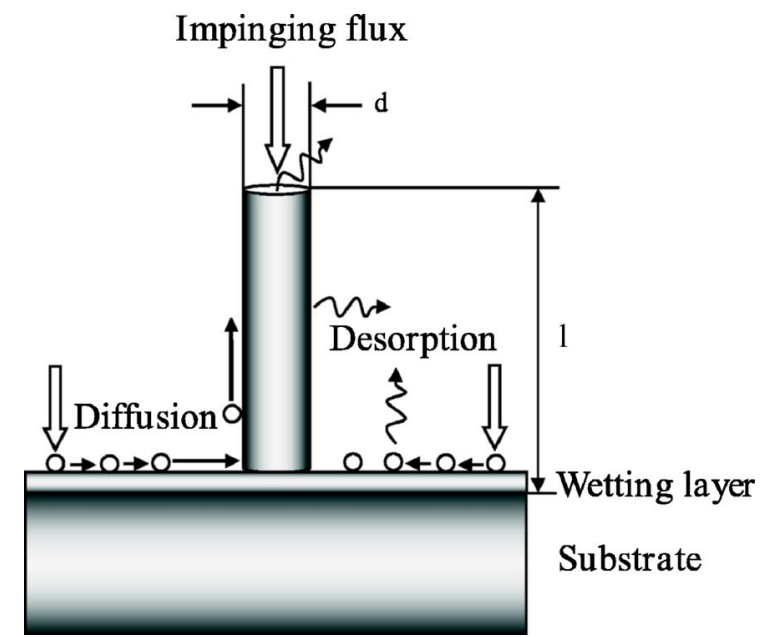

FIG. 1. (Color online) Schematic of the MBE growth process of NWs in which the relevant processes such as adsorption, desorption, diffusion, and nucleation are included.

grams of NW diameter distributions determined by the SEM for two samples with different growth times in the nucleation regime are shown in Fig. 2. We can see that by increasing the growth time from 30 to $60 \mathrm{~min}$, the most probable value of the diameter increases from 12 to $15 \mathrm{~nm}$.

For deposition times longer than $60 \mathrm{~min}$, we assume the nucleation to be finished and a few wires coalesce into thicker ones, while many others continue to grow separately. We have investigated the correlation between the length and diameter of NWs for a long deposition time of $360 \mathrm{~min}$. This correlation is shown in Fig. 3. The NW length decreases with diameter, similar to the catalyst-assisted MBE growth of GaAs (Refs. 13 and 17) and Si (Ref. 18) NWs. For thinner

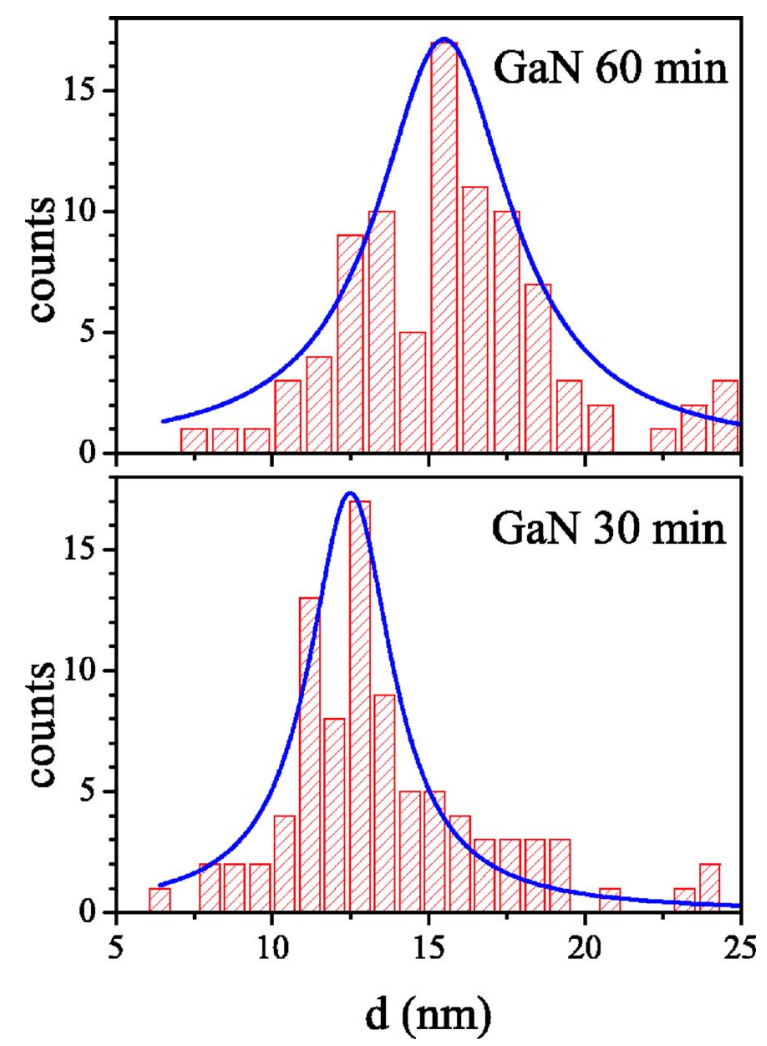

FIG. 2. (Color online) Histograms showing the distribution of the NW diameter in the nucleation growth regime for growth times of 30 and $60 \mathrm{~min}$.

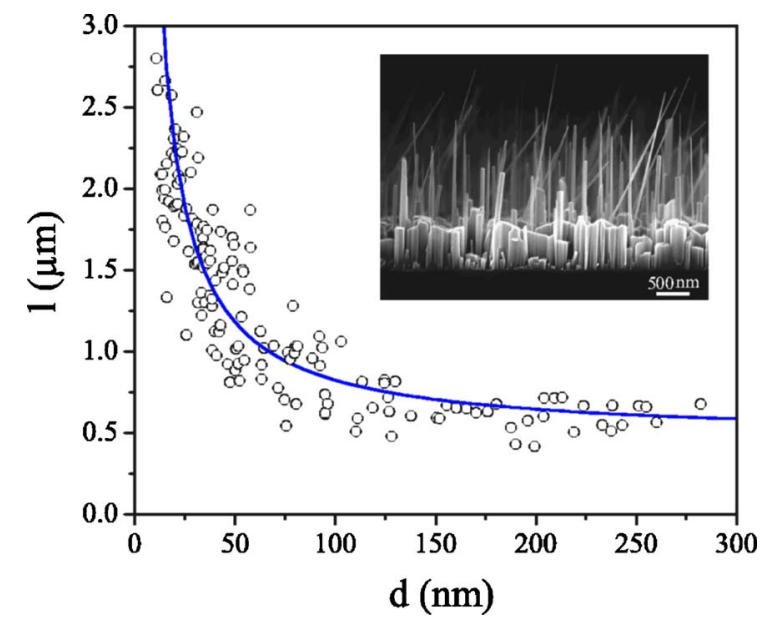

FIG. 3. (Color online) Length and diameter dependence of GaN NWs grown at $796^{\circ} \mathrm{C}$ for $360 \mathrm{~min}$ with Ga BEP of $3 \times 10^{-8} \mathrm{mbar}$. The data are fitted according to relation (1). The inset shows the corresponding SEM image.

wires, an aspect ratio (length/diameter) of about 250 was achieved. Some of the longer wires grow tilted relative to the $\mathrm{Si}$ substrate. For the same diameter, the tilted wires are longer than the normal ones and are not included in the data of Fig. 3. Such a growth enhancement was also observed by Givargizov $^{19}$ and is attributed to a stepped surface of the alternative growth face, which reduces the activation energy of the NW growth.

There is a general agreement in literature ${ }^{17,18}$ that the observed length/diameter dependence can be explained by a kinetic process including the diffusion of adatoms on the substrate surface and side faces of the wires, as schematically shown in Fig. 1. Growth proceeds in the DI regime if the diffusion flux of the adatoms from the sidewalls to the top is comparable to the deposition rate. Kinetic models, which explain the NW growth in this diffusion regime, have been published with earlier ${ }^{20}$ and recent investigations. ${ }^{13}$ Most of the models show a linear dependence of the wire length $(l)$ versus reciprocal diameter $(1 / d)$ for longer deposition time,

$$
l=C_{1}\left(1+C_{2} / d\right),
$$

where $C_{1}$ and $C_{2}$ are constants strongly related to the analyzed sample. A fit of this formula to the experimental data in Fig. 3 gives the values of 470 and $80 \mathrm{~nm}$ for $C_{1}$ and $C_{2}$, respectively. The asymptotic value $C_{1}$ is related to the thickness of a compact layer. The second coefficient $C_{2}$ is related to the DI growth. With simplified conditions imposed in the theoretical model proposed by Johansson et al. ${ }^{4}$ the coefficient $C_{2}$ is two times the diffusion length, $\delta\left(C_{2}=2 \delta\right)$ along the side of the NW, and the growth of the thinner wires is limited by the diffusion process on sidewalls. The diffusion length on the wire surface is given by $\delta=(D \tau)^{1 / 2}$, where $D$ is the surface diffusion coefficient and $\tau$ is the stay time of an adatom at the surface before it is desorbed or incorporated. When $l>\delta$, atoms condensing at the base of the wire are no longer able to reach the top before desorption takes place (reevaporation of adatoms) and the rate of arrival of adatoms at the wire tip (impinging flux on top plus diffused adatoms from an $\sim \delta$ distance on sidewall) becomes constant with the growth time.

A diffusion length much shorter than the column length leads to a strong tapering effect. In our case, wires with a length of $2-3 \mu \mathrm{m}$ showed no pronounced tapering. Follow- 
ing this argument, we should conclude that with a diffusion length of about $40 \mathrm{~nm}$, the mechanism limiting the growth should not be related to a short diffusion length, which is underestimated due to simplified assumptions in the model of Johansson et al. ${ }^{4}$

Besides a possible limitation due to the diffusion process itself, other mechanisms might be involved. One of these is related to the barriers that adatoms have to overcome when jumping from one surface to another (see Fig. 1). Further investigations are necessary to distinguish between different mechanisms. Nevertheless, we can generally conclude that MBE growth of GaN NWs on $\mathrm{Si}(111)$ substrates under the growth conditions given above is dominated by DI growth below $d=80 \mathrm{~nm}\left(C_{2}\right)$. For larger diameters, direct impingement is the main contributing mechanism to the growth.

In conclusion, experimental results on GaN-NW growth for a deposition time, which exceeds the nucleation time, have been explained in the frame of the DI growth model. For thinner wires with diameters ranging from 10 to $80 \mathrm{~nm}$, growth is enhanced by adatom diffusion on sidewalls, while for thicker wires this additional adatom flux is less important and only the adsorption at the tip of the wire plays an important role. This gives rise to short thick NWs as well as thin long ones with a very high aspect ratio. An aspect ratio of about 250 has been achieved for longer deposition time. An approximately linear dependence of NW length as a function of reciprocal of the diameter has been observed in agreement with the DI mechanism.

The authors would like to thank K.-H. Deussen for technical support and H. P. Bochem for the SEM analysis. One of the authors (R.K.D.) appreciates the financial support from a Helmholtz-DAAD Fellowship.

${ }^{1}$ A. B. Greytak, C. J. Barrelet, Y. Li, and C. M. Lieber, Appl. Phys. Lett. 87, 151103 (2005).

${ }^{2}$ F. Qian, S. Gradecak, Y. Li, C. Y. Wen, and C. M. Lieber, Nano Lett. 5, 2287 (2005).

${ }^{3}$ Y. Cui and C. M. Lieber, Science 291, 851 (2001).

${ }^{4}$ J. Johansson, C. P. Svensson, T. Martensson, L. Samuelson, and W. Seifert, J. Phys. Chem. B 109, 13567 (2005).

${ }^{5}$ R. Meijers, T. Richter, R. Calarco, T. Stoica, H. P. Bochem, M. Marso, and H. Lüth, J. Cryst. Growth 289, 381 (2006).

${ }^{6}$ T. Stoica, R. Meijers, R. Calarco, T. Richter, and H. Lüth, J. Cryst. Growth 290, 241 (2006).

${ }^{7}$ L. E. Jensen, M. T. Björk, S. Jeppesen, A. I. Persson, B. J. Ohlsson, and L. Samuelson, Nano Lett. 4, 1961 (2004).

${ }^{8}$ R. S. Wagner and W. C. Ellis, Appl. Phys. Lett. 4, 89 (1964).

${ }^{9}$ K. Haraguchi, K. Hiruma, M. Yazawa, and T. Katsuyama, J. Electrochem. Soc. 153, C1 (2006).

${ }^{10}$ V. G. Dubrovskii, I. P. Soshnikov, N. V. Sibirev, G. E. Cirlin, and V. M. Ustinov, J. Cryst. Growth 289, 31 (2006).

${ }^{11}$ J. Johansson, B. A. Wacaser, K. A. Dick, and W. Seifert, Nanotechnology 17, S335 (2006).

${ }^{12}$ V. G. Dubrovskii, N. V. Sibirev, G. E. Cirlin, J. C. Harmand, and V. M. Ustinov, Phys. Rev. E 73, 021603 (2006).

${ }^{13}$ V. G. Dubrovskii, G. E. Cirlin, I. P. Soshnikov, A. A. Tonkikh, N. V. Sibirev, Yu. B. Samsonenko, and V. M. Ustinov, Phys. Rev. B 71, 205325 (2005).

${ }^{14}$ V. Mamutin, Tech. Phys. Lett. 25, 741 (1999).

${ }^{15}$ K. A. Bertness, A. Roshko, N. A. Sanford, J. M. Barker, and A. V. Davydov, J. Cryst. Growth 287, 522 (2006).

${ }^{16}$ E. Calleja, M. A. Sánchez-García, F. J. Sánchez, F. Calle, F. B. Naranjo, E. Muñoz, U. Jahn, and K. Ploog, Phys. Rev. B 62, 16826 (2000).

${ }^{17}$ M. C. Plante and R. R. Lapierre, J. Cryst. Growth 286, 394 (2006).

${ }^{18}$ L. Schubert, P. Werner, N. D. Zakharov, G. Gerth, F. M. Kolb, L. Long, and U. Gösele, Appl. Phys. Lett. 84, 4968 (2004).

${ }^{19}$ E. I. Givargizov, J. Cryst. Growth 31, 20 (1975).

${ }^{20}$ V. Ruth and J. P. Hirth, J. Chem. Phys. 41, 3139 (1964). 